\title{
Propose with a Rose? \\ Signaling in Internet Dating Markets
}

\author{
Soohyung Lee \\ University of Maryland, College Park and MPRC \\ Muriel Niederle \\ Stanford University and NBER \\ Hye-Rim Kim and Woo-Keum Kim \\ Korea Marriage Culture Institute
}

\begin{abstract}
A growing theoretical literature on market design studies the impact of preference signaling on market, and several markets have a way to allow agents to signal their preferences, such as the US college application and the economics junior market. However, empirically evaluating the impact of preference signaling proves difficult due to data limitations. In this paper we use a randomized field experiment to provide evidence that a signaling system can affect matching outcomes in real matching environments. In our experiment of online dating events, "virtual roses" are used to signal a participant's special interest in another participant. We find that offers with roses are more likely to be accepted than offers without roses. Furthermore, participants with more roses get a larger number of dates than their counterparts.
\end{abstract}

JEL Classification:

Keywords: experiment, matching, signaling, market design, online dating 